\section{Utilisation de morphiniques épiduraux et} sous-arachnoidiens

\author{
Introduction \\ Efficacité \\ Douleur chronique \\ Douleur post-opératoire \\ Douleurs du travail et de l'accouchement \\ Effets secondaires \\ Dépression respiratoire \\ Autres effets secondaires \\ Considérations particulières \\ Sites d'action \\ Concentrations plasmatiques \\ Courbe dose-réponse \\ Choix du morphinique \\ Therapie de longue durée \\ Conclusions \\ Utilisation dans la douleur aiguë \\ Utilisation dans la douleur chronique
}

Peu de techniques ont rencontré l'approbation générale aussi rapidement que l'administration centrale de morphiniques. Dès que l'efficacité des morphiniques intrathécaux et épiduraux fut rapportee, cette méthode a connu une popularité immédiate grâce à son coût peu élevé, la simplicité de son administration et sa longue durće d'action. En revisant la littérature pertinente à l'analgésie morphinique épidurale et sous-arachnoïdienne, nous avions pour objectif d'offrir au lecteur une vue d'ensemble de l'expérience publiée sur cette question. Nous nous sommes permis de tirer certaines conelusions que nous n'avons pas la prétention de présenter comme les ultimes règles de l"art, mais comme des suggestions pouvant permettre au praticien l'utilisation plus rationnelle d'une technique un peu mieux connue.

\section{Effets bénéfiques}

\section{Douleur chronique}

La première expérience clinique portant sur l'utilisation sous-arachnoïdienne de morphiniques fut par Wang, ${ }^{1,2}$ et faisait suite à des études animales qui en avaient démontré l'efficacité. ${ }^{3}$ Cette étude portant sur huit patients souffrant de douleurs chroniques soulagés pendant 20 heures par 0.5 ou $1 \mathrm{mg}$ de morphine injectée dans l'espace sous-arachnoïdien sans effets secondaires fut suivie de plusieurs autres. L'approche épidurale fut bientôt tentée pour ce même type de douleur ${ }^{4,5}$ et on peut retenir de ces expériences que la morphine à la dose de 2 à $5 \mathrm{mg}$ dans un volume adequat, habituellement de $10 \mathrm{ml}$, était efficace (tableau 1); le soulagement dura plus longtemps avec la morphine qu'avec la mépéridine ou le fentanyl et la tolérance prit plus de temps à se manifester lorsque la morphine était mélangée à un anesthésique local.

\section{Douleur post-opératoire}

Par la suite on appliqua la méthode d'injection épidurale ou sous arachnoïdienne au soulagement de douleurs aigues, post-opératoires ou traumatiques. Des injections rachidiennes ${ }^{6-8}$ et plus fréquemment épidurales ${ }^{9-21}$ (tableau I) ont été utilisées pour le confort post-opératoire, sans qu'on ait à recourir à des injections systémiques additionnelles. Les doses de morphine rachidiennes ont varié de 0.2 à $2 \mathrm{mg}$ et épidurales, de 2 à $10 \mathrm{mg}$. Tous les types de chinurgie ont éprouvé l'efficacité de ces méthodes qui fut trouvée supérieure au placebo, ${ }^{9} \mathrm{de}$ plus longue durée que la bupivacainne ${ }^{14}$ et sans le retentissement cardiovasculaire ${ }^{15}$ de cette dernière. On a également fait ressortir l'importance d'injectcr le morphinique au niveau des segments médullaires

TABLEAU I Efficacité analgésique de la morphine épidurale et intrathécalc

\begin{tabular}{llll}
\hline & Posologie épidurale $(m g)$ & Posologie intrathécale $(m g)$ & Durée d'action (hr) \\
\hline Douleur chronique $^{(1-5)}$ & $2-5$ & $0.5-1$ & $6-24$ \\
Douleur post-opératoire $^{(6-27)}$ & $2-10$ & $0.2-2$ & $12-20$ \\
Douleur obstétricale $^{(28-4)}$ & $2-6$ & $1-2$ & $?$ \\
\hline
\end{tabular}


TABLEAU II Dépression respiratoire après morphine epidurale et intrathécale ${ }^{(44-45)}$

\begin{tabular}{llcc}
\hline Voie & Posologie(mg) & Retard(hr) & Incidence(\%) \\
\hline Intrathécale & $1-15$ & $4-11$ & $4-7$ \\
Epidurale & $2-20$ & $0.5-12$ & $0.25-0.4$ \\
\hline
\end{tabular}

appropriés ${ }^{22}$ de même que l'importance de la dose de morphine en rapport avec le type de douleur, ${ }^{22}$ bien qu'une injection épidurale lombaire haute se soit montrée efficace dans la chirurgie thoracique. ${ }^{23}$ Finalement, on a comparé l'injection épidurale à l'injection intramusculaire et au blocage intercostal conventionnel et on a démontré la supériorité de la morphine épidurale lorsqu'on mesurait la durée analgésique, ${ }^{24-27}$ qui pouvait varier de 12 à 20 heures selon l'étude, le type de chirurgie, ou la méthode d'évaluation utilisée.

Douleut du travail et de l'accouchement

Les promesses suscitées par une analgésie de longue durée, sans retentissement vasomoteur et sans bloc moteur, ont amené de nombreux cliniciens à utiliser des morphiniques sous-arachnoïdiens ou épiduraux en obstétrique ${ }^{28-43}$ (tableau I). Ceux qui ont utilisé 1 et $2 \mathrm{mg}$ de morphine par la voie sous-arachnoidienne rapportent un degré certain d'efficacité pendant le travail sauf lors de l'expulsion du bébé, ${ }^{29,31}$ alors que les injections épidurales se sont avérées inefficaces ${ }^{32,35}$ ou peu effica$\operatorname{ces}^{36-42}$ selon les auteurs. Un facteur étiologique souvent invoqué pour expliquer le peu d'efficacité des morphiniques épiduraux pour ce type de douleur est l'augmentation de la vascularisation de l'espace épidural chez les parturientes avec absorption rapide des morphiniques injectés à ce niveau. Ceci a conduit certains auteurs à ajouter de l'adrénaline à la solution. ${ }^{36,40,43}$ Malgré cette addition, on n'a noté que peu d'amélioration dans les résultats. Nous en concluons qu'actuellement les narcotiques épiduraux n'ont que peu d'utilité clinique en obstétrique.

\section{Effets secondaires}

\section{Dépression respiratoire}

Peu de temps après la publication des premiers résultats sur l'utilisation d'opiacés par voie sousarachnoïdienne et épidurale, on commençait à rapporter des effets secondaires, dont le plus sérieux était sans contredit la dépression respiratoire (tableau II). En effet, des cas de dépression respiratoire grave et de coma furent décrits après des injections de morphine sous-arachnoïdiennes administrées 4 à 11 heures auparavant, caractérisés par une bradypnée et un myosis extrêmes. ${ }^{44-46}$ Les doses utilisées avaient été de 1 à $15 \mathrm{mg} \mathrm{et}$, dans tous les cas, la naloxone à dose unique ou répétée a renversé la situation. L'incidence de dépression respiratoire se situe de 4 à 7 pour cent par la voie sous-arachnoïdienne et de 0.25 à 0.4 pour cent par la voie épidurale ${ }^{47}$ Cette dépression respiratoire a tendance à être précoce dans les 30 minutes qui suivent l'injection épidurale pour un morphinique lipophile comme le fentanyl (distribution octanol/ eau 11,220$)$, * la mépéridine (distribution octanol/ eau 38.82) la méthadone (distribution octanol/eau $116.33)^{64}$ ou tardive (4 à 12 heures après l'adminis. tration) pour un morphinique peu lipophile comme la morphine (distribution octanol/eau 1.42), 1'absorption plus rapide des agents lipophiles expliquant cette différence. Plusieurs autres facteurs viennent modifier l'importance de la dépression respiratoire comme la dose utilisée, l'association de morphiniques intra-musculaire, ${ }^{48}$ ]'âge du patient, la présence de pathologie associée de type apnée du sommeil, ${ }^{49}$ la position du malade après l'injection, ${ }^{50}$ l'accoutumance aux morphiniques. Quelques études complêtées par des courbes de réponse au $\mathrm{CO}_{2}$ et des pressions d'occlusion ${ }^{51-54}$ confirment le fait que des injections de morphine épidurale de $2 \mathrm{mg}$ et plus peuyent causer une dépression respiratoire tardive, même chez des patients non tarés. On en a conclu que tout patient qui reçoit en particulier de la morphine épidurale devrait être étroitement surveillé pour 12 à 24 heures après son administration.

\section{Autres effets secondaires}

Les autres effets secondaires rapportés sont plus fréquents mais moins dramatiques. Ce sont les nausées, le prurit et la rétention urinaire. Ces effets secondaires sont rapportés dans 15 à 70 pour cent des cas. ${ }^{55,56}$ En obstétrique, on a également rapporté la nécessité de hausser la dose d'oxytocine chez les patientes qui recevaient $2 \mathrm{mg}$ de morphine sous-arachnoïdienne par rapport à celles qui en

*Communication personnelle de Janssen Pharmaceutica Inc. 
TABLEAU III Morphine épidurale et intrathécale: concentrations plasmatiques

\begin{tabular}{|c|c|c|c|}
\hline Yoie & Posologie (mg) & Taux (ng) & Moment du prelevement (min) \\
\hline Intrathécale ${ }^{(74)}$ & $1-1.75$ & 6 & 120 \\
\hline Intrathécale $e^{(72)}$ & $0.2 / \mathrm{kg}$ & 42 & 60 \\
\hline Epidurale( & $0.2 / \mathrm{kg}$ & 67 & 60 \\
\hline Epidurale $^{(70)}$ & $0.05 / \mathrm{kg}$ & 19 & 12 \\
\hline Epidurale $^{(68)}$ & $5 / 70 \mathrm{~kg}$ & 43 & 15 \\
\hline Epidurale $^{(7)}$ & 4 & 12.5 & 20 \\
\hline
\end{tabular}

recevaient $1 \mathrm{mg} .{ }^{29}$ Ces effets secondaires semblent être plus marqués pour les posologies les plus élevées, une êtude ayant démontré que dans l'injection intrathécale de morphine les patients avec dose élevée présentaient plus de sédation, de nausées, de rétention urinaire ct de dépression respiratoire que ceux avec une dose moindre. ${ }^{57}$ Peu d'effets cardiovasculaires importants sont décrits, sauf ceux qui sont secondaires à la dépression respiratoire.$^{58} \mathrm{On}$ a également montré que l'hypoalgésie cutanée progressait en direction rostrale, et s'accompagnait d'une atteinte du trijumeau plusieurs heures après l'injection épidurale. ${ }^{59}$ Par ailleurs, les morphiniques épiduraux ne semblent pas avoir d'effet sur le débit sanguin utérin. ${ }^{60}$ Un cas de catatonie après des injections épidurales répétés de morphine a été rapporte. ${ }^{61}$ Il ne semble pas que la morphine sans préservatif diluée dans du soluté physiologique produise des lésions de démyélinisation, d'arachnoidite ou de nécrose. ${ }^{62}$

\section{Considérations particulières}

\section{Sites d'action}

L'action des morphiniques injectés dans l'espace sous-arachnoïdien ou épidural semble reliée à la présence de récepteurs opiacés dans les lamina 1,2 et 5 de la corne dorsale de la substance grise de la moëlle épinière. ${ }^{63}$ L'injection de morphiniques a confirmé cette hypothèse chez l'animal ${ }^{3}$ et chez l'humain. $^{1,2} \mathrm{~A}$ des doses de $10 \mathrm{mg}$ de morphine épidurale, on a pu démontrer une hypoalgésie segmentaire à expansion céphalique au site d'injection et qui atteint le territoire du trijumeau après 6-9 heures. ${ }^{64,65}$ Cette progression rostrale est surtout evidente avec les morphiniques peu liposolubles comme la morphine et peut expliquer certains effets secondaires comme le prurit, la dépression respiratoire, les nausées et vomissements, qui sont le fait d'une interaction entre le narcotique et les centre nerveux. La mesure de la concentration de morphinique dans le liquide céphalorachidien a d'ail. leurs prouvé le passage rapide de la mépéridine, en particulier, de l'espace épidural au liquide céphalorachidien. ${ }^{66,67}$ Le fait qu'un antagoniste des morphiniques, le naloxone, bloque tous les effets secondaires comme les nausées, le prurit, la rétention urinaire et la dépression respiratoire montre que les morphiniques se lient à des sites spécifiques dont ils peuvent être déplacés. La nécessité d'administrer des doses répétées de naloxone pour dissiper des effets secondaires qui récidivent après un certain temps démontre le lien solide qui s'établit entre le morphinique et son récepteur.

\section{Concentrations plasmatiques}

Après une injection épidurale, les concentrations plasmatiques maximales de morphine étaient atteintes de 20 à 30 minutes plus tard ${ }^{68-71}$ (tableau III) La dépression respiratoire précoce qui survient au mème moment semble donc être en relation directe avec la concentration plasmatique, contrairement à la dépression tardive qui survient après 90 minutes. ${ }^{68}$ Cette dissociation, selon certains, confirme l'action analgésique spinale directe et segmentaire. ${ }^{68,70,71}$ Une étude au cours de laquelle on a administré $0.2 \mathrm{mg} \cdot \mathrm{kg}^{-1}$ de morphine épidurale, sous-arachnoïdienne et intra-musculaire, a montré que la concentration sérique de morphine était la plus élevée après l'injection intramusculaire, suivie de l'injection épidurale et de l'injection sousarachnoïdienne, mais qu'il $y$ a très peu de différence entre la voie intra-musculaire et la voie épidurale. ${ }^{72}$

Par ailleurs, il semble qu'il y ait un passage rapide de l'espace épidural de la mère au placenta, ${ }^{73.74}$ ce qui pourait représenter un risque pour le fœtus. Quoiqu'il en soit, après une injection épidurale, la concentration de morphiniques dans le 
liquide céphalo-rachidien est de 40 à 100 fois plus élevée que dans le sang ${ }^{75}$ et il y a production d'une hypoalgésic intense et prolongée. Il semble donc que cette analgésie de longue durée soit en relation avec les concentrations de morphiniques dans le liquide céphalorachidien et non pas avec les concentrations plasmatiques, ${ }^{23}$

\section{Courbe dose-réponse}

Que ce soit par la voie sous-arachnoïdienne ou épidurale, au début les doses de morphine ou d'autres morphiniques ont été administrées de façon empirique et variaient beaucoup. On s'est vite rencu compte que les complications et leur sévérité pouvaient être en relation directe avec la dose et que possiblement on pourrait obtenir un effet thérapeutique satisfaisant avec des doses moindres et ce avec moins d'effets secondaires. Plusieurs études portant surtout sur l'analgésie épidurale qui est devenue la voie la plus populaire, ont comparé entre elles les différentes doses de morphiniques administrées à des malades comparables. ${ }^{76-78}$

Les conclusions qu'on peut tirer de ces études sont les suivantes: pour l'adulte, dans la chirurgic abdominale, une dose de 4 ou $5 \mathrm{mg}$ de morphine épidurale est tout aussi efficace et moins susceptible d'entrainer des complications que des doses plus élevées dc 8 ou $10 \mathrm{mg}^{76,77}$ Toutefois des doses moindres de l'ordre de $2 \mathrm{mg}$ par exemple sont moins efficaces. Pour une chirurgie du membre inférieur, une dose de $2 \mathrm{mg}$ est aussi efficace que des doses de 4 et $8 \mathrm{mg}$, et produit moins d'effets secondaires $^{22,32,78}$ (tableau IV). Lorsqu'on administre un morphinique par cette voie, une posologie basée sur le poids ne semble pas appropriée: '13 chez l'enfant, dans une publication sur les injections caudales de morphine post-interventions génitales on a utilisé une dose de morphine de $0.5 \mathrm{ml} \cdot \mathrm{kg}^{-1}$ d'une solution de $0.1 \mathrm{mg} \cdot \mathrm{ml}^{-1}$ avec succès et sans complications. ${ }^{26}$

\section{Choix du morphinique}

Mépéridine, fentanyl, morphine, méthadone, Betaendorphine ont tous été utilisés et comparés par la voie épidurale ou sous-arachnoïdienne ${ }^{79-81}$ Il en ressort toujours que la morphine a la plus longue durée d'action et est tout aussi efficace que les autres morphiniques. Des mélanges de morphiniques et d'autres médicaments ont aussi été administrés. Ainsi, il semble qu'expérimentalement
TABLE IV Morphine épidurale: courbes dose-réponse

\begin{tabular}{|c|c|c|}
\hline Écude & Type de chirurgie & Dose suggérée \\
\hline \multirow[t]{2}{*}{ Rawal N. et al..$^{(22)}$} & $\begin{array}{c}\text { abdominale et } \\
\text { thoracique }\end{array}$ & 4 \\
\hline & orthopedique & 2 \\
\hline Chayen M.S. et al. ${ }^{(32)}$ & orthopedique & 2 \\
\hline Martin R. et al. ${ }^{(78)}$ & orthopédique & 2 \\
\hline Carmichael F.J (74) & abdominale & 4 \\
\hline Crawford R.D. et al. ${ }^{(76)}$ & abdominale & 5 \\
\hline
\end{tabular}

le dropéridol prolonge la durée d'action de la morphine intrathécale et potentialise son effet, tout en diminuant le phénomène de tolérance. ${ }^{82}$ De plus, l'addition d'adrénaline à la solution morphinique diminuerait les taux sériques de morphine, tout en augmentant la vitessc d'action et en prolongeant la durée, ${ }^{83}$ mais cette conclusion ne fait pas l'unanimité. ${ }^{4,71}$ Des mélanges d'anesthésiques locaux et de morphine ont également été injectés et il ne semble pas y avoir d'interaction entre ces médicaments. 13,43

\section{Thérapie prolongée}

L'usage de morphinique par voie épidurale et sous-arachnoïdienne a évolué vers l'administration prolongée chez des patients souffrant de néoplasies ou de douleurs chroniques réfractaires. On a d'abord commencé par laisser des cathéters dans l'espace épidural pour de longues périodes et d'injecter par intermittences; ; ${ }^{84-87}$ les résultats ont été bons avec les premières injections mais des signes de tolérance sont apparus après une dizaine de jours, ce qui nécessita une augmentation de la dose. L'étape ultérieure a été l'implantation permanente de cathéters épiduraux et sous-arachnoidiens branchés sur différents types de réservoir ou de pompes à perfusion. ${ }^{88.89}$ Ces implantations ont donné des résultats encourageants, mais ont conduit à une tachyphylaxie sans dépression respiratoire, ce qui pourrait être le résultat d'une tolérance progressive aux morphiniques lorsque des concentrations constantes de morphine sont maintenues dans le liquide céphalo-rachidien. ${ }^{89}$

On s'est demandé si l'administration épidurale ou sous-arachnoïdienne répétée de morphiniques pouvait être cause de neurotoxicité: dans deux cas, à l'autopsie, les cordons postérieurs ont montré des signes de dégénérescence qui pourrait être causée par l'implantation prolongée du cathéter de silas- 
TABLEAU V Morphiniques épiduraux: utilisation dans la douleur aiguë

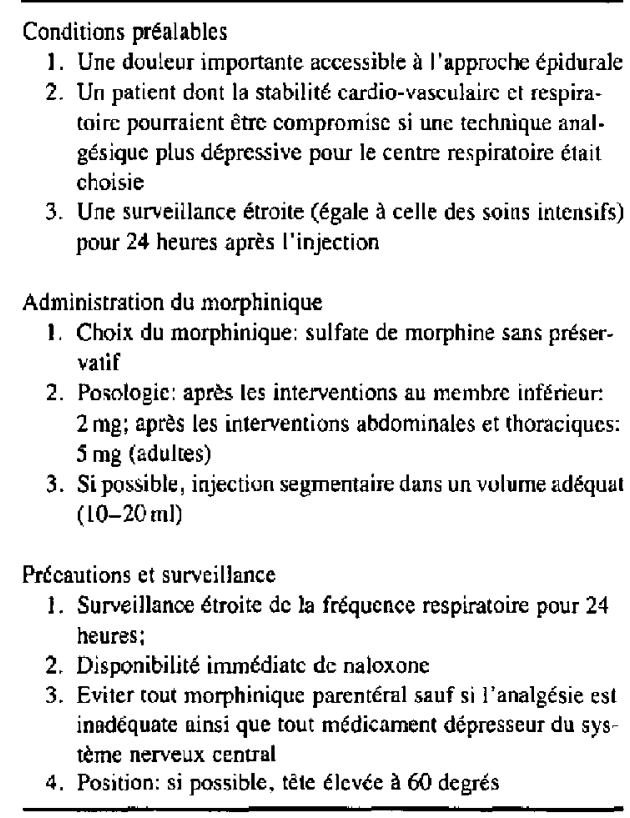

tic, ${ }^{90,91}$ des opiacés ou par la pathologie sousjacente elle-même.

\section{Conclusions}

Utilisation dans la douleur aiguë

L'efficacité des morphiniques épiduraux ou sousarachnoidiens semble maintenant bien démontrée. Malgré des avantages certains sur d'autres modes d'analgésie, les effets secondaires limitent actuellement la genéralisation de la technique et on ne devrait par conséquent l'utiliser que dans des cas choisis. ${ }^{75,92,93}$ Certaines conditions doivent être remplies avant l'injection épidurale ou sous-arachnoïdienne et la modalité d'administration doit être conforme à certaines normes dictées par nos connaissances actuelles. La voie épidurale nous semble actuellement celle qui présente le meilleur rapport coût/bénéfice et à moins de contre-indication elle devrait être préférée à la voie sous-arachnoïdienne. ${ }^{70}$ C'est donc pour cette voie que nous proposons des modalités d'administration (tableau V). Le domaine obștêtrical, bien que théoriquement intéressant, ${ }^{93}$ se prête mal à ce type d'analgésie comme en fait foi la majorité des expériences cliniques. Les Betaendorphines pourraient cependant trouver une place en obstétrique. ${ }^{94}$

\section{Utilisation dans la douleur chronique}

L'utilisation de morphiniques épiduraux ou sousarachnoidiens pour la douleur chronique d'après les expériences cliniques n'est pas exempte d'inconvénients causés par la tolérance et la tachyphylaxie. Ceci restreint le nombre de candidats à l'installation de cathéters sous-arachnoïdiens ou épiduraux permanents à des patients dont l'espérance de vie est très limitée. Chez certains patients répondant à ce critère et non soulagés par des traitements conventionnels, cette technique nous semble une alternative valable compte tenu du fait qu'ils présentent déjà une tolérance marquée et sont donc peu sujets à des complications respiratoires. Un test d'efficacité est obligatoire avant l'insertion permanente de ces cathéters.

\section{References}

1 Wang $J F$. Soulagement de la doulcur par injcction intrathécale de sérotonine ou de morphine. Ann Anesth Franc 1978; XIX: 371-2.

2 Wang $J F$, Nauss LA, Thomas JE. Pain relief by intrathecalty applied morphine in man. Anesthesiology 1979; 50: 149-51.

3 Yarsh $T L$, Rudy TA. Analgesia medicated by a direct spinal action of narcotics. Science 1976; 192: 1357-8.

4 Behar $M$, Magora $F$, Olshwang $D$, Davidson JT. Epidural morphine in treatment of pain. Lancet 1979; 1: 527-8.

5 Stoyanov M, Muller H, Hempelmann G. Traitement des douleurs chroniques, utilisation des morphiniques par la voie peridurale. Anesth Anal Réan 1981; 38: 375-8.

6 Tung A, Maliniak $K$, Tenicela $R$, Winter $P$. Intrathecal morphine for intraperative and postoperative analgesia. JAMA 1980; 244: 2637-8.

7 Decastro J, D'Imverno E, Lecron L, et al. Prespectives d'utilisation de morphinoïdes en anesthésie loco régionale. Anesth Analg Réanim 1980; 37: 17-24

8 Gjessing J, Tomlin PJ. Postoperative pain control with intrathecal morphine. Anaesthesia 1981 ; 36: $268-76$. 
9 McClure JH, Chambers WA, Moore E, Scott DB. Epidural morphine for postoperative pain. Lancet 1980; 975-6.

10 Redick $L F$, Bromage PR. Postpartum epidural narcotic analgesia. Anesthesiology 1980; 53: S297.

11 Yu M, Youngstrom PC, Cowan RI, et al. Postcesarian epidural morphine: double blind study. Anesthesiology 1980; 53: S216.

12 Magara $F$. Olswang $D$, Eimerl $D$, et al. Observations on extradural morphine analgesia in various pain conditions. Br J Anaesth 1980; 52: 247-52.

13 Lecron L, Toppet-Ralatoni E, Rogaerts J. Essais comparatifs des différentes techniques antalgiques par voie médullaire. Anesth Anal Réan 1980; 37: $549-56$.

14 Graham JL, King R, McCaughey W. Postoperative pain relief using epidural morphine. Anaesthesia 1980; 35: 158-60.

15 Johrston JR, McChughey W. Epidural morphine, a method of management of multiple fractured ribs. Anacsthesia 1980; 35: 155-7.

16 Modig J. Paalzow L. A comparison of cpidural morphine and epidural bupivacaine for postoperative pain relief. Acta Anesthesiol Scand $1981 ; 25: 437-41$.

17 El-Baz NM, Ganzouri AR, GotschalkW, et al. Thoracic epidural analgesia for pain relief after thoracic surgery. Anesthesiology 1982; 57: A-205.

18 Dailey PA, Kotelko DM, Shnider $S M_{r}$ et al. Effectiveness and safety of epidural morphine for postoperative pain after Cesarean section. Anesthesiology 1982; 57: A-387.

19 Allen PD, Walman AT, Cullen SDJ, et al. The effects of epidural morphine on postoperative analgesia. Anesthesiology 1982; 57: A199.

20 Shapiro LA, Hoffman $S$, Jedeikin $R$, Kaplan $R$. Single injection epidural anesthesthesia with bupivacaine and morphine for prostatectomy. Anesth Analg 1981; 60: 818-20.

21 Jensen BH. Caudal block for postoperative pain relief in children after genital operations. A comparison between bupivacaïne and morphine. Acta Anaesthesiol Scand 1981; 25: 373-5.

22 Rawal N, Sjostrand V, Dahlastrom B. Postoperative pain relief by epidural morphine. Anesth Analg 1981 60: 726-31.

23 Nordberg $G$, Hedner $T$, Mellstrand T, et al. Pharmacokinetic aspects of epidural analgesia. Ancsthesiology 1983; 58: 545-51.

24 Lanz $E$, Theiss $D$, Reiss W, Sommer $V$, Epidural morphine for postoperative analgesia: A doubleblind study. Anesth Analg 1982; 61: 236-40.

25 RawalN, Sjostrand VH, Dahlstrom B, et al. Epidural morphine for postoperative pain relief: A comparative study with intramuscular narcotic and intercostal nerve block. Anesth Analg 1982; 61: 93-8.

26 Jensen PJ, Siem-Jorgensen P, Neilsen TB, ei al. Epidural morphine by the caudal route for postoperative pain relief. Acta Anaesthesiol Scand 1982;26: 511-3.

27 Rybro L, Schurizek BA, Petersen TK, Wernberg $M$. Postoperative analgesia and lung function: A comparison of intra-muscular with epidural morphine. Acta Anaesthesiol Scand 1982; 26: 514-8.

28 Scott PV, Bowen FA, Cartwright P, ef al. Intrathecal morphine as sole analgesic during labor. Br Med J 1980; $281: 351-5$.

29 Baraka $A$, Noueihid $K$, Hajj $S$. Intrathecal injection of morphine for obstetric analgesia. Anesthesiology $1981 ; 54: 136-40$.

30 Srinivasan $T$. Intrathecal morphine for obstetric analgesia. Anesthesiology 1981; 55: A298.

31 Abboud TK, Shnider SM, Dayley PA, et al. Intrathecal morshine for the relief of labor pain. Anesthesiology 1982; 57 : A384.

32 Chayen MS, Rudick V, Borvine A. Pain control with epidural injection of morphine. Anesthesiology 1980; 53: 338-9.

33 Husemeyer RP, $O^{\prime}$ ConnorMC, Davenport $H T$. Failure of epidural morphine to relieve pain in labour. Anaesthesia 1980; 35: 161-3.

34 Crawford JS. Experience with epidural morphine in obstetrics. Anaesthesia 1981; 36: 207-9.

35 Writer WDR, James FM, Wheeler A5. Double-blind comparison of morphine and bupivacaine for continuous epidural analgesia in labour. Ancsthesiology 1981; 54: 21.5-219.

36 Booker $P D$, Wilkes RG, Bryson THL, Beddard J. Obstetric pain relief using epidural morphine. Anaesthesia 1980; 35; 377-9.

37 Perriss $B W$. Epidural pethidine in labour. Anaesthesia 1980; 35: 380-2.

38 Magara F, Danchin Y, Olswang D, Schenker JG. Epidural morphine analgesia in second-trimester induced abortion. Am J Obst Gynccol 1980; 138: 260-2.

39 Muller A, Laugner B. Farcot JM, er al. Hypoalgésic obstctrical par injection péridurale de morphine. Anesth Anal Réan 1981; 38: 35-41.

40 Skjoldebrand A, Garle M, Gustafsson L, et al. Ex- 
tradural pethidine with and without adrenaline during labour: wide variation in effect. $\mathrm{Br} \mathrm{J}$ Anaesth 1982; 54: 415-20.

41 Justins DM, Francis D, Houlton PG, Reynolds $F$. A controlled trial of extradural fentanyl in labour. Bri J Anaesth 1982; 54: 409-14.

42 Baraka A, Maksabi M, Houeihid R. Epidural meperidine-bupivacaine for obstetrical analgesia. Anesth Analg 1982; 61: 652-6.

43 Martin R. Tétrault JP, Lamarche Y, et al. Epidural morphine for obstetrical analgesia: The effect of epinephrine, Can Anacsth Soc J 1983; 30: S72.

44 Glynn CJ, Mather RLE, Causins MJ, et al. Spinal rlarsotics and respiratory depression. Lancet 1979; 11: $356-7$.

45 Lialios A, Andersen FF. Selective spinal analgesia. Lancet 1979; 11: 357.

46 Davies GK, Tollhurst-Cleaver $C L$, James $T L$. CNS depression from intrathecal morphine. Anesthesiology $1980 ; 52: 580$.

47 Gustafssan $L L$, Schildt B, Jacobsen K. Adverse effects of extradural and intrathecal opiates: report of a nationwide survey in Sweden, Br J Anaesth 1982; 54: 479-86.

48 Gustafsson $L L$, Feychting $B$, Klingstent $C$. Late respiratory depression after concomitant use of morphine epidurally and parenterally, Lancet 1981; 892-3.

49 Lamarche $Y$, Martin $R$, Blaise $G$. Severe respiratory depression after epidural morphine in a patient with sleep apnea. Submitted for publication.

50 McCaughey W, Granham JL. The respiratory depression of epidural morphine. Anaesthesia 1982; 37: $990-5$.

51 Doblar DD, Mulduon SM, Abbrecht PH, et al. Epidural morphine following epidural local ancsthesia: effect on ventilatory and airway occlusion pressure to $\mathrm{CO}_{2}$. Anesthesiology 1981; 55: 423-8.

52 Holland $R B$. Carbon dioxide response after epidural morphine. Anaesthesia 1982; 37: 753-7.

53 Knill RL, Clement JL, Thompson WR. Epidural morphine causes delayed and prolonged ventilatory depression. Can Anaesth Soc J 1981; 28: 537-43.

54 Neilsen CH, Camporesi EM, Bromage PR, et al. $\mathrm{CO}_{2}$ sensitivity after epidural and I.V. Morphine. Anesthesiology 1981; 55: A372.

55 Mok MS, Tsai SK. More experience with intrathecal morphine for obstetric analgesia. Anesthesiology $1981 ; 55: 481$
56 Reiz $S$, Wesiberg $M$. Side-effects of epidural morphine. Lancet 1980; 203-4.

57 Samii $K$, Chauvin $M$, Viars $P$. Postoperative analgesia with morphine. Br J Anaesth 1981; 53: 817-20.

58 LiaoJC, Harrison P, Buckley JJ, Takemori A. Sympathetic reflexes in morphine vs lidocaine spinal block. Anesthesiology, 1981; 55: A148.

59 Bromage PR, Camporesi EM, Durant PAD, Neilsen $\mathrm{CH}$. Nonrespiratory side effects of epidural morphine. Anesth Analg 1982; 61: 490-95.

60 Rosen MA, Hughes SC, Curtis JD, et al. Effects of epidural morphine on uterine blood flow and acidbase status in pregnant ewe. Anesthesiology 1982; 57: A383.

61 Engquist $A$, Jopgensen $B C$, Andersen $H B$. Catatonia after epidural morphine. Acta Anaesthesiol Scand $1981 ; 25: 445-6$.

62 Abouleish E, Barmado MA, Nemeto EM, et al Acute and chronic effects of intrathecal morphine in monkeys. Anesthesiology 1981; 55: A153.

63 Kitahata LM, Kosaka Y, Taub A, et al. Laminaspecific suppression of dorsal-horn activity by morphine sulfate. Anesthesiology 1974; 41: 39-48.

64 Bromage $P R$, Camporesi EM, Durant PA, Neilsen $\mathrm{CH}$. Rostral spread of epidural morphine. Anesthesiology 1982; 56: 431-6.

65 Thompson WR, Smith PT, Hirst $M$, et al. Regional analgesic effect of epidural morphine in volunteers. Can Anaesth Soc J 1981; 28: 530-6.

66 Cousins MJ, Macher LE, Glynn CJ, er al. Selective spinal analgesia. Lancet 1979; 1: 1141-2.

67 Glynn CJ, Mather LE. Cousins MI, et al. Peridural meperidine in humans: Analgesic response, pharmacokinetics, and transmission into CSF. Anesthesiology $1981 ; 55$ : 520-6.

68 Weddel S, Ritter R. Serum levels following epidural administration of morphine and correlation with relief of postsurgical pain. Anesthesiology $1981 ; 54$ : 210-4.

69 Moller IW, Vester-Andersen T, Steentoft A, et al. Respiratory depression and morphine concentration in serum after epidural and intramuscular administration of morphine. Acta Araesthesiol Scand 1982; 26: 421-4.

70 Gustafsson $L L$, Friberg-Wielsen $S_{r}$ Garle $M_{r}$ et al Extradura] and parenteral morphine: Kinetics and effects in postoperative pain. A controlled clinical study. Br J Anaesth 1982; 54: 1167-74.

71 Yougnstrom PC, Cowan RI, Sutheimer C, et al. Pain 
relief and plasma concentrations from epidural and intramuscular morphine in post-cesarean patients. Anesthesiology 1982; 57: 404-7.

72 Chauvin M, Samii K, Schermann JM, et al. Plasma concentrations of morphine after I.M., extradural and intrathecal administration. Br J Anaesth 1981; 53: $911-3$.

73 Nybell-Lindahl G, Carlsson C, Ingemarsson I, et al. Maternal and fetal concentrations of morphine after epidural administration during labor. Am J Obstet Gynecol 1981; 139: 20-21.

74 Bonnardot JP, Maillet $M$, ColauJC, et al. Maternal and fetal concentration of morphine after intrathecal administration during labour. Br J Anaesth 1982; 54 : 487-9.

75 Bromage PR. Intraspinal narcotics: State of the art. Refresher course in anesthesiology 1982;10:27-36.

76 Crawford RD, Batra MS, Fox F. Epidural morphine dose response for postoperative analgesia. Anesthesiology 1981; 55: A150.

77 Carmichael FJ, Rolbin $S H$, Hew EM. Epidural morphine for analgesia after Cacsarian section. Can Anaesth Soc J 1982; 29: 359-63.

78 Martin R, Salbaing J, Blaise G, et al. Epidural morphine for postoperative pain relief: A dose-response curve. Anesthesiology 1982; 56: 423-6.

79 Rutter DV, Skewes G, Morgan M. Extradural opioids for postoperative analgesia: A double-blind comparison of petidine, fentanyl and morphine. $\mathrm{Br} J$ Anaesth 1981; 53: 915-29.

80 Oyama T, Fukushi S, Jin T. Epidural B-endorphin in treatment of pain. Can Anaesth Soc J 1982; 29: 24-6.

81 Torda TA, Pybus DA. Comparisan of four narcotic analgesics for extradural analgesia. $\mathrm{Br} J$ Anaesth 1982; 54: 291-5.

$82 \mathrm{Kim} K C$. Stoelting $R F$. Effect of droperidol on the duration of analgesia and development of tolerance to intrathecal morphine. Anesthesiology 1980; 53: $\$ 219$.

83 Bromage PR, Camporesi EM, Durant PAC, Neilsen $\mathrm{CH}$. Influence of epinephrine as an adjuvant to epidural morphine. Anesthesiology 1983; 58: 25762.

84 Howard RP, Milne LA, Williams NE. Epidural morphine in terminal care. Anaesthesia 1981;36: 51-53.

85 McCoy DD, Miller MG. Epidural morphine in a terminally ill patient. Anesthesiology 1982; 57: 427. 86 Zenz M, Schappler-Scheele P, Neuhaus R, er al.
Long-term peridural morphine analgesia in cancer pain. Lancet 1981; 91.

87 Wishart JM. Epidural morphine at home. Can Anaesth Soc J 1981; $28: 492$.

88 Coombs DW, Saunders RL, Pageau MG. Continuous intraspinal narcotic analgesia. Technical aspects of an implantable infusion system. Regional Anesth $1982 ; 7 ; 110-3$.

89 Coombs DW, Saunders RL, Gaylor M, Pageau MG. Epidural narcotic infusion reservoir: Implantation technique and efficacy. Anesthesiology 1982; 56 : 469-73.

90 Meier FA, Coombs DW, Saunders RL, Pageau MG Pathologic anatomy of constant morphine infusion by intraspinal silastic catheter. Anesthesiology 1982 ; 57: A206.

91 Abouleish E, Barmada MA, Nemolo EM, et al. Acute and chronic effects of intrathecal morphine in monkeys. Br J Anaesth 1981; 53: 1027-32.

92 Klinck $J R$, Lindop $M J$. Epidural morphine in the elderly. Anaesthesia 1982; 37: 907-12.

93 Wyant GM. Déjà vu (éditorial). Can Anaesth Soc J 1981; 28: 521-2.

94 Oyama $T$, Matsuki A, Taneighi $T$, et al. Betaendorphin in obstetric analgesia. Am J Obstet Gynecol $1980 ; 137$. 613-6. 